\title{
HPV genotyping: need of the hour for cervical cancer screening
}

\author{
Poonam Sharma, Ashish Khanna, Sarbjeet Sharma, Jasvir Kaur \\ Department of Microbiology, Sri Guru Ram Das Institute of Medical Sciences and Research (SGRDIMSAR), Amritsar, Punjab, India
}

\begin{abstract}
Objectives: Cervical cancer is the most common cancer and leading cause of cancer deaths in women in developing countries. India shares about one-fourth of the global burden of cervical cancer and almost all cases harbour high risk Humanpapilloma virus (HPV) infection. This is a unique cancer in the sense that it is totally attributable to the effects of an infectious virus. Therefore, the present study was undertaken to evaluate the distribution of HPV genotypes in a rural area of Punjab, with the aim of identifying the most frequent HPV genotypes associated with different cervical lesions in this population.
\end{abstract}

Materials and Methods: The study was conducted over a period of two years i.e. from January2013 to January 2015 , on women attending obstetrics and gynaecology department of a tertiary care hospital. One hundred sixty consecutive scrape samples or cervical brushings were collected from women presenting with any type of cervical lesions. DNA was extracted from the clinical sample using Hybribio DNA extraction kit. Hybribio HPV genoarray test kit was used to genotype the HPV risk groups.

Results: Out of 160 cervical brushings collected over a period of two years, $17(10.6 \%)$ were positive for HPV genotypes. Among the latter, HPV18 genotype was observed in 7(41.17\%), HPV16 genotype in 3 (17.65\%) and HPV53 genotype in $2(11.76 \%)$. One of the genotypes $68,52,39,33$ and 15 were present in rest of the samples.

Conclusion: HPV genotypes 18 followed by 16 were the most common genotypes found in the present study. HPV vaccination along with HPV based screening may go a long way in controlling cervical cancer in India. J Microbiol Infect Dis 2016;6(4): 168-171

Key Words: HPV, Cervical cancer, Screening, Genotyping

\section{INTRODUCTION}

Human Papilloma Virus (HPV) is the commonest sexually transmitted disease agent worldwide presenting clinically as genital warts or existing asymptomatically in men and women [1]. Its association with genital tract malignancies in general, and etiological and necessary factor of cervical cancer of women in particular has been the main interest in this double stranded DNA virus of family Papillomaviridae.

Cervical cancer is the second most cancer in women all over the world [2]. This is a unique cancer in the sense that it is totally attributable to the effects of an infectious virus. India shares about 25 per cent of global cervical cancer burden and almost all cases harbour high risk HPV infection [3].

HPVs have been divided into more than 200 genotypes based on DNA sequences, approximate- ly 80 of which have been well characterized. Genotypes have differing cellular tropism resulting in association of HPV types with specific clinical types of warts. Presently 14 types have been characterized as oncogenic and 3 (i.e., HPV 26, 73, and 82) have been characterized as possibly oncogenic $[4,5]$. The prevalence of HPV is dependent of the geographical region, but HPV 16 is by far the most prevalent and oncogenic HPV type [6]. Typically HPV18, 45, 31 and 33 are the next most prevalent types but the order varies between geographical areas. In other areas, such as Asia, HPV58 and HPV52 are the next most common after HPV16 and HPV18 [7].

Overall prevalence of HPV in cervix is $10 \%$ higher in women of developing countries. HPV infection is most common in sexually active young females but cervical cancer is common in older women suggesting infection at younger age and slow progression to cancer [8]. 
However, HPV genotype, persistent infection, co-infection, number of sexual partners, age, parity, long term use of oral contraceptives are important factors which will determine whether or not a woman infected with HPV will develop cervical cancer [8].

So the present study was undertaken to evaluate the distribution of HPV genotypes in a rural area of Punjab, with the aim of identifying the most frequent HPV genotypes associated with different cervical lesions in this population.

\section{MATERIALS AND METHODS}

The study was conducted over a period of two years i.e. from January2013 to January 2015, on women attending obstetrics and gynaecology department of a tertiary care hospital. One hundred sixty consecutive scrape samples or cervical brushings were collected from women presenting with any type of cervical lesions such as chronic cervicitis, endocervicitis, cervical erosions, cervical laceration, cervical polyp, leukoplakia of cervix and basal cell hyperplasia of cervix.

The cervical brushings collected from patients were stored at $4^{\circ} \mathrm{C}$ after collection, to be processed within 2 weeks.

Patients who had intercourse $24 \mathrm{hrs}$ prior to the test and patients during menstrual period were excluded from the study. Hybribio human papilloma virus genoarray test kit was used which uses the combination of both polymerase chain reaction and flow through hybridisation technology for the qualitative detection and determine the specific HPV type present by genotyping 21 types of HPV DNA in cervical specimens. It can genotype the following HPV risk groups:

HighRisk:HPV16,18,31,33,35,39,45,51,52,56, $58,59,66,68$

\section{Low Risk: HPV 6, 11,42,43,44}

\section{Undetermined Risk: HPV 53, CP8304}

The assay was performed according to the manufacturer's protocol. DNA was extracted from the clinical sample using Hybribio DNA extraction kit. PCR was performed with a reaction volume of $25 \mu \mathrm{l}$ containing $1 \mu \mathrm{l}$ of DNA template, $23.25 \mu \mathrm{l}$ of PCR mix and $.75 \mu$ l of DNA Taq polymerase in a applied bio systems Perkin Elmer 9600, GeneAmp PCR system 9700,PTC-200,EPPENDORF master thermal cycler.
The amplification protocol was as follows: 9 min of denaturation at $95^{\circ} \mathrm{C}$, and 40 cycles of 20 s of denaturation at $95^{\circ} \mathrm{C}, 30$ s of annealing at $55^{\circ} \mathrm{C}$ and $30 \mathrm{~s}$ of elongation at $72^{\circ} \mathrm{C}$, followed by final extension for $5 \mathrm{~min}$ at $72^{\circ} \mathrm{C}$. Then it was incubated at $4^{\circ} \mathrm{C}$. The amplicon were subsequently denatured and subjected to hybridisation. The assay utilises a flow through hybridisation technique by actively directing the targeting molecules towards the immobilized probes within the membrane, with the complementary molecules being retained by the formation of duplexes. After a stringent wash, the hybrids were detected by the addition of streptavidin-horseradish peroxidase conjugate (provided with the kit), which binds to the biotinylated PCR products, and a substrate (nitro blue tetrazolium-5-bromo-4-chloro-3-indolylphosphate) to generate a purple precipitate at the probe dot. The results were interpreted using direct visualisation.

After hybridisation, the presence of positive result for both the "internal control" and the "biotin" dots within the membrane indicated that the isolated DNA was of good quality, the enzyme conjugate was valid, and the hybridisation process was proper. Demographic and clinical data of the patients with cervical lesions was recorded.

\section{RESULTS}

Out of 160 cervical brushings collected over a period of two years, 17 (10.6\%) were positive for HPV genotypes. Among the latter, HPV18 genotype was observed in 7 (41.17\%), HPV16 genotype in 3 (17.65\%) and HPV53 genotype in 2(11.76\%). One of the genotypes $68,52,39,33$ and 15 were present in rest of the samples. Most commonly found HPV genotypes found in our study i.e. HPV 18 and 16 , were the ones, which fall under high-risk group. Other than genotype 53 and 15 which are categorised under undetermined group, rest of the genotypes viz. 33, 39, 52, 68 andd 33 observed in this study also belong to high risk group. Among these 17 positive patients, $12(70.6 \%)$ were in the age group of 30-40yrs. However all the females with genotype 18 belonged to the age group of 30-40, two women with HPV genotype 16 were in 50-60 age group and one was in 30-40 age group. Rest of them were in 20-30 age groups. After going through their demographic profile as shown in Tables 1, 2, 3 and 4 , it was noticed that urban or rural background, literacy status and parity perhaps had no association with HPV positivity. 
Table 1. Distribution \& HPV positivity of patients according to their backgrounds

\begin{tabular}{lccc}
\hline Background & No. Of patients & HPV +ve & HPV -ve \\
\hline Rural & $98(61.2 \%)$ & $9(9.2 \%)$ & $89(91 \%)$ \\
Urban & $62(38.7 \%)$ & $8(13 \%)$ & $54(87 \%)$ \\
Total & 160 & $17(10.6 \%)$ & $143(89.3 \%)$ \\
\hline
\end{tabular}

Table 2. Distribution \& HPV positivity of patients according to their education

\begin{tabular}{lccc}
\hline Education & No. Of Patients & HPV +ve & HPV -ve \\
\hline $\begin{array}{l}\text { Below or up to } \\
10^{\text {th }} \text { standard }\end{array}$ & 16 & $2(12.5 \%)$ & $14(87.5 \%)$ \\
$10+2$ standard & 73 & $5(6.8 \%)$ & $68(93.2 \%)$ \\
$\begin{array}{l}\text { Graduate and } \\
\text { above }\end{array}$ & 71 & $10(14 \%)$ & $61(86 \%)$ \\
Total & 160 & 17 & 143 \\
\hline
\end{tabular}

Table 3. Distribution \& HPV positivity of patients according to their parity

\begin{tabular}{lccc}
\hline Parity & No. Of Patients & HPV +ve & HPV -ve \\
\hline One child & 8 & $2(25 \%)$ & $6(75 \%)$ \\
Two & 129 & $15(11.6 \%)$ & $114(88.4 \%)$ \\
More than two & 23 & - & $23(100 \%)$ \\
Total & 160 & 17 & 143 \\
\hline
\end{tabular}

Table 4. Distribution \& HPV positivity of patients according to their age group

\begin{tabular}{lccc}
\hline Age Group & No. Of Patients & HPV +ve & HPV -ve \\
\hline Less than 30yrs & 17 & $1(5.9 \%)$ & $16(94.1 \%)$ \\
$30-40 y r s$ & 96 & $12(12.5 \%)$ & $84(87.5 \%)$ \\
$41-50$ & 37 & $2(5.4 \%)$ & $35(94.6 \%)$ \\
$51-60$ & 10 & $2(20 \%)$ & $8(80 \%)$ \\
Total & 160 & 17 & 143 \\
\hline
\end{tabular}

\section{DISCUSSION}

Persistent infection with high-risk HPV types is an important cause of cervical cancer $[9,10]$. However, early detection and subsequent effective treatment of HPV in precancerous lesions can prevent progression to cancer [11]. The present study shows that high risk genotypes 16 and 18 were present in females with cervical lesions which is in agreement with other studies [12,13]. High risk genotype 18 was the most common genotype in this study however in the study by Basu $P$ et al genotype 16 was the most common [14]. They also found co-infection by genotypes 16 and 18 . In present study no coinfection was observed. Genotypes 16, 18, 31, 33, and 45 were the five most common types, detected in $87.1 \%$ of the total cases in the study by Basu $\mathrm{P}$ et al, however in the present study most commonly detected genotype was 18 , followed by 16,53 and 33 , constituting $70.6 \%$ of the total cases. HPV types 16 and 18 are consistently the two most common types in invasive cancer, globally.

The highest proportion of positive HPV cases $(15 / 17,88.2 \%)$ in our study corresponded to patients younger than 40 years, which was also observed by Morelva Toro in his study [15]. In the present study parity had no association with HPV infection which is in accordance with other studies $[12,16]$. The main limitation of our study is that these observations relied on a small number of cases. However, our findings will contribute to HPV knowledge in rural area of Punjab, India that will be useful for enforcing implementation of vaccine use and to augment screening strategies. Our data suggest that a vaccine targeting HPV genotypes 18 and 16 can help prevent cervical cancers in this region. The study by Munoz $\mathrm{N}$ et al suggested that vaccination against HPV16 and 18 could prevent almost $70-80 \%$ of invasive cervical cancers worldwide [17]. As the sensitivity and specificity of cytology-based screening is not optimum, and there is low screening coverage in most parts of India, immunization against the most prevalent high risk HPV genotypes affecting each region may represent the most effective means to long-term cervical cancer prevention [18].

HPV genotyping can be used as a primary screening method to select patients at risk of disease, but in resource poor settings this will not be feasible unless HPV screening is made cheaper. In summary, cervical cancer screening is now entering a new era in which we will increasingly rely on measuring the causative virus and its associated high risk HPV genotypes rather than the pleomorphic cellular changes caused by the infection.

A progress from cytology-based screening to HPV-based screening may prove useful in stratifying HPV positive women according to risk of developing prevalent precancerous and cancerous lesion, to determine the appropriate clinical management strategy.HPV testing has recently been investigated as an alternative to two diagnostic modalities (cytology and colposcopy) for the detection of persistent or recurrent disease. If HPV DNA is undetectable 6 to 8 months post treatment, the likelihood of post- 
treatment persistence or recurrence of disease is negligible.

In conclusion, for establishing effective preventive measures it is prudent to determine the types of HPV genotypes most commonly associated with cervical cell transformation in different geographical areas ,as the current strategies for the prevention of cervical cancer and their precancerous lesions are based on the HPV genotyping and prophylactic vaccines [19-22].

HPV vaccination along with HPV based screening may go a long way in controlling cervical cancer.

Declaration of Conflicting Interests: The authors declare that they have no conflict of interest.

Financial Disclosure: No financial support was received.

\section{REFERENCES}

1. Burd EM. Human papilloma virus and cervical cancer. Clin Microbiol Rev 2003; 16:1-17.

2. Shanta V, Krishnamurthi S, Gajalakshmi CK, et al. Epidemiology of cancer of the cervix: global and national perspective. J Indian Med Assoc.2000; 98:49-52.

3. Speich N, Schmitt C, Bollmann R, et al. Human Papilloma Virus study of 2916 cytological samples by PCR and DNA sequencing: genotype spectrum of patients from west German area. J Med Microbiol 2004; 53:125-128.

4. Munoz N, Bosch FX, de Sanjose S, et al. Epidemiologic classification of human papillomavirus types associated with cervical cancer. N Engl J Med 2003;348:518-527.

5. Cogliano V, Baan R, Straif K, Grosse Y, Secretan B, El Ghissassi $F$. Carcinogenicity of human papillomaviruses. Lancet Oncol 2005;6:204.

6. Clifford GM, Gallus S, Herrero R, et al. Worldwide distribution of human papillomavirus types in cytologically normal women in the International Agency for Research on Cancer HPV prevalence surveys: a pooled analysis. Lancet 2005; 366:991-998.

7. Clifford GM, Smith JS, Plummer M, et al. Humanpapillomavirus types in invasive cervical cancer worldwide: a metaanalysis.Br J Cancer 2003;88:63-73

8. MolanoM, Van den Brule A, Plummer M, et al. HPV study group. Determinants of clearance of human papillomavirus infections in Colombian women with normal cytology: a population based, 5 years follow-up study. Am J Epidemiol 2003; 158:486-494.
9. Walboomers JM, Jacobs MV, Manos MM, et al. Human papilloma virus is necessary cause of invasive cervical cancer worldwide. J Pathol 1999; 189:12-19.

10. Munoz N, Bosch FX, de Sanjose S, et al. International agency for research on cancer. Multicenter Cervical cancer study group. Epidemiological classification of human papilloma virus types associated with cervical cancer. N Engl J Med 2003; 348:518-527.

11. Spitzer M. Cervical screening adjuncts: recent advances. Am.J.Obstet. Gynecol 1998; 179:544-556.

12. Duttagupta C, Sengupta S, Roy M, et al. Are Muslim women less susceptible to oncogenic human papillomavirus infection. A study from rural eastern India. Int J Gynaecol Cancer 2004; 14:293-303.

13. Aggarwal R, Gupta S, Nijhawan R, et al. Prevalence of high -risk human papillomavirus infections in women with benign cervical cytology: a hospital based study from North India. Indian J. Cancer 2006; 43:110-116.

14. Basu P, Roychowdhury S, Bafna UD, et al. Human papillomavirus genotype distribution in cervical cancer in India: results from a multi-centre study. Asian Pac J Cancer Prev 2009; 10:27-34.

15. De Mendez MT. Prevalence of Human Papillomavirus (HPV) Genotypes and Multiple Infections in Routine Cervical Cancer Screening in a Spanish Regional Population. SOJ Microbiol Infect Dis 2013;1 (1):6.

16. Y.Lazcano-Ponce E, Herrero R, Munoz N, et al. Epidemiology of HPV infection among Mexican women with normal cervical cytology. Int J Cancer 2001; 91:412-420.

17. Munoz N, Bosch FX, Castellsague X, et al. Against which human papillomavirus types shall we vaccinate and screen? The international perspective. Int J Cancer 2004, 111:278285.

18. Cuzick J, Arbyn M, Sankaranarayanan R, et al. Overview of human papillomavirus-based and other novel options for cervical cancer screening in developed and developing countries. Vaccine 2008, 265:K29-K41.

19. Saslow D, Solomon D, Lawson HW, et al. ACS-ASCCP-ASCP Cervical Cancer Guideline Committee. American Cancer Society, American Society for Colposcopy and Cervical Pathology, and American Society for Clinical Pathology screening guidelines for the prevention and early detection of cervical cancer. Cancer J Clin 2012; 62(3):147-172.

20. Trottier H, Franco EL. Human papillomavirus and cervical cancer: burden of illness and basis for prevention. Am J Manag Care 2006; 12: S462-S472.

21. Franco EL, Cuzick J. Cervical cancer screening following prophylactic humanpapillomavirus vaccination. Vaccine 2008; 26: A16-A23.

22. No JH, Kim MK, JeonYT, Kim YB, Song YS. Human papillomavirus vaccine: widening the scope for cancer prevention. Mol Carcino 2011; 50(4): 244-253. 